\title{
Braunau e la casa natale di Hitler: un'eredità pesante
}

Nell'ottobre 2016 l'allora Ministro dell'Interno austriaco, Wolfgang Sobotka, ha dichiarato in un'intervista che la casa natale di Adolf Hitler a Braunau am Inn sarebbe stata abbattuta. La sua dichiarazione è il culmine di una lunga disputa, da anni aperta in Austria, sull'uso di quella casa. Una polemica che rivela non solo la difficoltà nella gestione dell'eredità pesante del nazionalsocialismo e dei suoi monumenti in Austria, ma anche la mancanza di una linea ufficiale su un passato controverso.

In October 2016, the Austrian Minister of the Interior, Wolfgang Sobotka, stated in an interview that Adolf Hitler's birthplace in Braunau am Inn was to be torn down. That statement was merely the pinnacle of a long conflict within Austrian public opinion that involves a larger picture than just the future use of this house. The tiresome history of these debates reflects not only the difficulties the Austrian State faces with the remnants of the national-socialistic past and it's influence today, but also the lack of an official line about this controversial past.

\section{Introduzione}

Nell'ottobre 2016 l'allora Ministro dell'Interno austriaco, Wolfgang Sobotka, ha dichiarato in un'intervista che la casa natale di Adolf Hitler sarebbe stata abbattuta. La sua dichiarazione è il culmine di una lunga disputa attorno all'uso di quella casa che da anni è in corso in Austria. Questa accesa discussione rivela non solo le difficoltà nella gestione dell'eredità pesante del nazionalsocialismo e dei suoi 
monumenti, ma anche il problema di trovare una linea chiara con il passato controverso austriaco.

\section{Adolf Hitler e la sua casa natale}

La storia di Braunau am Inn oggi è collegata inevitabilmente con la figura di Adolf Hitler, che qui nacque il 20 aprile 1889. La città si trova nella regione dell'Oberösterreich, lungo il fiume Inn che rappresenta anche il confine con la Baviera. La casa dove il futuro Führer vide la luce in una stanza del secondo piano si trova nel quartiere Salzburger Vorstadt ed è oggi famosa in tutto il mondo, anche se Hitler visse solo per un breve periodo in città: solo poche settimane dopo la nascita la famiglia lasciò la casa e tre anni dopo anche Braunau. Hitler tornò solo una volta nella sua vita a Braunau, il 12 marzo 1938, giorno dell'Anschluss con la Germania, quando attraversò il confine proprio vicino alla città per andare a Linz, che amava di più [Baumhack1 2015, 13].

Braunau e la casa natale quindi non giocarono mai un ruolo fondamentale nella vita di Hitler e - al contrario di Predappio - non vennero utilizzate per la creazione di un mito delle origini del Führer, se non per qualche tentativo propagandistico a livello locale. Negli anni della Erste Republik (Prima repubblica) la casa fu adibita, come già avvenuto in precedenza, a trattoria. Dopo l'Anschluss, la Nsdap l'acquistò per 150.000 Reichsmark dal proprietario Josef Pommer. L'intenzione era quella di trasformarla in un luogo che «ogni tedesco deve visitare almeno una volta nella vita», come affermava la Neue Warte am Inn, giornale locale di Braunau, riadattando il secondo piano com'era nel 1889 con ritratti e altri utensili del giovane Hitler'. Tuttavia si trattava di un'iniziativa regionale non coordinata con la propaganda centrale del Terzo Reich, per cui solo nel 1943, in occasione del cinquantaquattresimo compleanno di Hitler, la casa venne aperta al pubblico per «motivi di educazione popolare e culturale», con una biblioteca e una mostra d'arte ${ }^{2}$. Non venne realizzato però l'annunciato "luogo della memoria" e neanche Hitler partecipò alle manifestazioni. 


\section{Post-1945: tra damnatio memorie e prime iniziative}

Dopo la Seconda guerra mondiale la casa venne affidata al comune di Braunau, ma la vedova di Josef Pommer sporse querela contro l'amministrazione comunale e nel 1954 gli eredi ne riottennero la proprietà ${ }^{3}$ Le vicende successive mostrano bene le difficoltà che la nuova repubblica austriaca aveva con la pesante eredità del passato.

La linea ufficiale fu caratterizzata dalla damnatio memoriae. L'Austria si dichiarava come prima vittima del nazionalsocialismo, sulla base della Dichiarazione di Mosca del 1943 con la quale gli alleati l'avevano riconosciuta come vittima della politica di aggressione di Hitler [Liebhart 2007, 477]. La Dichiarazione divenne colonna portante dell'Opermythos ("mito della vittima"), che per molti anni rimase la posizione ufficiale dei governi austriaci fornendo alla società la possibilità di ricominciare senza fare i conti con il proprio passato [Judt 2002, 168].

Braunau rimaneva un problema perché si trattava di una località particolare. Nonostante fosse tornata di proprietà privata, negli anni Sessanta la casa fu utilizzata dal comune come sede scolastica e biblioteca pubblica ${ }^{4}$. Negli anni Settanta il Comune si preoccupò che gruppi di neonazisti potessero affittare la casa e chiese sostengo al governo del Partito socialdemocratico austriaco (SPÖ) sotto la guida del cancelliere Bruno Kreisky 5 . Nel 1972 la Repubblica austriaca divenne così locataria. Nel contratto d'affitto venne vietato, dopo lunghe discussioni con la proprietaria, ogni uso in "contesto storico-contemporaneo", escludendo così anche un eventuale utilizzo museale. Nel 1974 entrò in scena l'Associazione assistenziale Lebenshilfe che aprì un centro per disabili e rimase locatoria della casa fino al 2011, quando l'abbandonò a causa delle barriere architettoniche (l'adeguamento fu impedito dalla proprietaria Gerlinde Pommer) [Baumhackl 2015, 20-21]. Una più matura riflessione critica sul passato prese il via nella seconda parte degli anni Ottanta, con l'abbandono del "mito della vittima" e il riconoscimento del ruolo di responsabilità da parte dell'Austria per i crimini del nazionalsocialismo. Una data importante in tal senso è rappresentata dal discorso dell'allora Canceliere Franz Vranitzky, tenuto 1'8 luglio 1991 davanti al parlamento austriaco [Uhl 2001, 26].

Si veda il documento originale del processo di restituzione del 1954 in http://braunau-history.at/w/images/6/6b/ Rueckstellungsvergleich1.jpg.

Zöchling C., Das Geisterhaus, «Profil», 10 novembre 2014.

Ibidem. 
Questo processo, proseguito fino a oggi, è il prodotto soprattutto di varie iniziative a livello nazionale, regionale e comunale, che hanno reso la "cultura della memoria" (Erinnerungskultur) una componente centrale della memoria collettiva austriaca. Il caso di Braunau mostra bene i cambiamenti politico-culturali avvenuti a partire degli anni Ottanta, anche se le iniziative, nel caso specifico, furono prese non dagli organi istituzionali centrali ma a livello regionale. Gli anni Ottanta rappresentarono così l'inizio di diverse attività con lo scopo di rendere visibile l'eredità pesante della città. Già nel 1983 il Consiglio comunale di Braunau voleva installare una targa commemorativa alla facciata della casa con il testo «Mai più fascismo - Ricordare milioni di morti». La proprietaria dell'immobile tuttavia si oppose per vie legali e dopo un processo l'apposizione della targa venne impedita in riferimento al contratto d'affitto del $1972^{6}$.

Un ulteriore tentativo venne effettuato nel 1989, quando il comune, nel centenario della nascita di Hitler, mise una lapide davanti alla casa, questa volta sul terreno comunale per evitare nuovi problemi con la proprietaria. Sulla lapide, proveniente dall'ex campo di concentramento di Mauthausen, distante circa 70 $\mathrm{km}$ da Braunau, furono incise le parole «Per pace, libertà e democrazia. Mai più fascismo. Ricordare milioni di morti» [Baumhackl 2015, 20]. Più di quarant'anni dopo la fine della Seconda guerra mondiale, la casa riceveva così per la prima volta un segno distintivo in riferimento alla sua storia.

Una successiva iniziativa si registrò nel 1992, con la Braunauer ZeitgeschichteTage ("Giornata della storia contemporanea di Braunau"), che dalla prima edizione ogni anno porta a Braunau storici e politologi per discutere su vari argomenti. Il tema prescelto per la prima edizione (25-27 settembre 1992) riguardava l'Unerwünschtes Erbe ("eredità indesiderata"), secondo un'idea del politologo Andreas Maislinger, anche fondatore del Gedenkdienst ("Servizio austriaco della memoria"), sorto sempre nel 1992. Insieme al giornalista Erich Marschall e ad altri collaboratori locali, Maislinger organizzò questa giornata di dibattito, invitando anche i rappresentanti di altre città con un'eredità difficile come Mauthausen, Gori e Predappio'.

Nel 2000, in collaborazione col giornale locale «Braunauer Rundschau» e con Reinhold Klika, lo stesso Maislinger è stato tra i promotori della campagna Braunau setzt ein Zeichen ("Braunau dà un segno"), lanciando l'idea di trasformare la casa in un "luogo di riconciliazione". Tutti i partiti del consiglio comunale di Braunau, incluso anche il partito di destra Fpö (che all'epoca governava a livello 
nazionale insieme con i conservatori Övp) sostennero l'iniziativa, promuovendo una raccolta di firme e chiedendo che la casa venisse comprata e trasformata ${ }^{8}$. Tuttavia la risposta del governo non fu chiara: mentre la vice-cancelliera Susanne Ries-Passer e il Ministro dell'Interno Ernst Strasser espressero sostegno al progetto, il cancelliere Wolfgang Schüssel e altri membri del governo glissarono?. Mancando, di nuovo, un esplicito appoggio politico per procedere con tale progetto, il destino della casa rimase indeciso.

\section{Demolizione ed espropriazione: la questione della casa rimane attuale}

Negli ultimi anni il dibattito sulla casa natale di Hitler ha rappresentato un argomento all'ordine del giorno sia per l'amministrazione comunale, sia per i governi centrali. Da quando la Lebenshilfe ha lasciato l'edificio nel 2011, lo stabile è vuoto. Il nuovo sindaco di Braunau, Johannes Waidbacher, ha cambiato più volte idea sul suo possibile uso, da luogo per attività culturali ad abitazione pubblica - come ha dichiarato in un'intervista del 2012, una soluzione che il sindaco però nel frattempo non sostiene più ${ }^{10}$. Nel 2014 la proprietaria Gerlinde Pommer ha annunciato una probabile vendita della casa. Per evitare che l'edificio passasse in mani di individui con posizioni revisioniste, la Repubblica austriaca è entrata in trattative con la proprietaria e ha formulato un'offerta nel giugno 2015, ma le trattative sono naufragate (secondo Pommer, non ci sono state nemmeno seri tentativi, come ha rivelato una persona di fiducia nel 2016 a un giornale locale) [Schöndorfer 2018, 115].

Nel frattempo, l'allora Ministro dell'Interno Johanna Mickl-Leitner ha incaricato la Interdisziplinäre Kommission zum verantwortungsvollen Umgang mit NSKultstätten ("Commissione interdisciplinare per un rapporto responsabile con luoghi di culto del nazionalsocialismo"), composta da storici, politologi e giuristi. Nel rapporto finale pubblicato nel novembre 2015, la commissione ha consigliato una profonda ristrutturazione della casa, con lo scopo di cambiarne totalmente

8 Geburtshaus Hitlers als Stätte der Versöhnung, "Der Standard», 10 febbraio 2000; Versöhnungsstätte in Hitlers Geburtshaus?, «Der Spiegel», 9 febbraio 2000.

9 Auch Minister für Gedenkstätte, «Der Kurier», 3 marzo 2000; Braunau setzt ein Zeichen, «Braunauer Rundschau», 24 febbraio 2000.

10 Hitler-Geburtshaus, «News», 20 settembre 2012.

11 R.J. Laglstorfer, Hier wurde doch unser Führer geboren, «Beirkssundschau», 24 ottobre 2016. 
la riconoscibilità. Inoltre, la commissione ha proposto un uso in chiave sociale o amministrativo (per esempio, come sede di forze dell'ordine o di pronto soccorso). Un'altra commissione, la Nutzungskommission ("Commissione per l'uso") composta dal presidente della regione Alta Austria e dal sindaco di Braunau, ha dichiarato che avrebbe favorito un futuro uso della casa per un'organizzazione di pubblica utilità [Schöndorfer 2018, 116]. Viceversa, nell'autunno 2016 il nuovo Ministro dell'Interno, Wolfgang Sobotka, si è dichiarato favorevole alla demolizione, provocando ulteriori polemiche' ${ }^{12}$.

Tuttavia, fallite le trattative con la proprietaria Pommer, il governo nel 2016 ha deciso di preparare un'apposita legge federale per l'espropriazione dell'immobile, nel cui testo si definiscono le case di nascita dei dittatori come «luoghi emotivamente molto carichi e perciò di particolare interesse per la società» ${ }^{13}$. Proprio per queste particolarità il parlamento austriaco il 14 dicembre 2016 ha votato a favore dell'espropriazione della casa natale di Hitler con i voti di quasi tutti i partiti. Nonostante il ricorso dell'ormai ex proprietaria, la Corte suprema ha deciso il 30 giugno 2017 che l'espropriazione è legittima poiché si tratta di luogo d'interesse pubblico e «il rifiuto senza compromessi del nazionalsocialismo è una parte integrante della Repubblica austrica $\gg^{14}$.

\section{Linee guida per l'uso}

Trascorsi due anni dall'espropriazione, la casa natale di Hitler a Braunau è ancora vuota. Anche il cambio di governo - con il partito di destra Fpö che ha sostituito i socialdemocratici della Spö come partner del partito conservatore Övp - ha rallentato le decisioni. Un membro del consiglio comunale di Braunau, l'esponente dei verdi David Stögmüller, ha consegnato una richiesta ufficiale al nuovo Ministro dell'Interno Herbert Kickl (Fpö) per chiedere l'avanzamento dei piani

0. Kronsteiner, Denkmalschützer wollen Hitler-Haus erhalten, «Der Standard», 20 settembre 2016; V. Wagener, Adolf Hitlers Geburtshaus wird abgerissen, «DW», 17 ottobre 2016; Abriss von Hitlers Geburtshaus ist vom Tisch, «Die Presse», 15 dicembre 2016.

\footnotetext{
Il testo della legge è accessibile nel sito parlamento austriaco: https://www.parlament.gv.at/PAKT/VHG/XXV/I/I_01250/ fname_547916.pdf.

Si vedano la decisione della Corte Suprema nella comunicazione stampa pubblicata il 30 giugno 2017, https://www. vfgh.gv.at/medien/Enteignung_von_Hitlers_Geburtshaus.de.php, e il testo della decisione della Corte Suprema, https:// www.ris.bka.gv.at/Dokument.wxe?Abfrage=Vfgh\&Dokumentnummer=JFT_20170630_17G00053_00. All'ex proprietaria è stato riconosciuto un risarcimento, considerato tuttavia troppo modesto, per cui è ancora aperta una vertenza giudiziaria tra la Pommer e lo stato austriaco: Hitler-Geburtshaus: Enteignung deutlich teurer als gedacht?, «Tiroler Tageszeitung», 21 giugno 2018.
} 
per il futuro uso. Il sottosegretario del Ministero dell'Interno, in un'intervista dell'aprile 2018, ha dichiarato che al momento sono in atto trattative con la Lebenshilfe e che si vuole fare un bando di gara per una profonda ristrutturazione architettonica. Lo storico Kim Christian Priemel dell'Università di Oslo ha proposto in un articolo sul giornale tedesco «Frankfurter Allgemeine» un "museo di Hitler" dove non venga ricostruita la biografia del Führer ma il suo impatto sulla vita comunitaria. Un simile progetto non sarebbe però possibile secondo il sindaco di Braunau Waidbacher, perché una musealizzazione è contro la legge sull'espropriazione'. Intanto l'Iniative Denkmalschutz ("Iniziativa per la tutela dei monumenti") ha sottolineato che dopo l'espropriazione non esistono più vincoli e ha quindi appoggiato l'idea della Haus der Verantwortung ("Casa della responsabilità") di Andreas Maislinger ${ }^{16}$.

Questa idea risale all'iniziativa del 2000 Braunau setzt ein Zeichen e prevede la creazione di un luogo d'incontro internazionale, con workshop e conferenze non solo su questioni storiche ma anche sui principi di libertà e diritti umani. Il focus sul passato rappresenta perciò solo una parte del progetto della "Casa della responsabilità". Ogni piano sarebbe dedicato a una diversa tematica. Il piano terra si occuperebbe del passato e di come interpretare la storia e gli avvenimenti del passato in generale (non solo, cioè, del passato di Braunau). Al primo piano sarebbe riservato spazio alla comprensione del presente, nel tentativo di formare una coscienza per i problemi e i conflitti attuali. Il secondo piano sarebbe invece

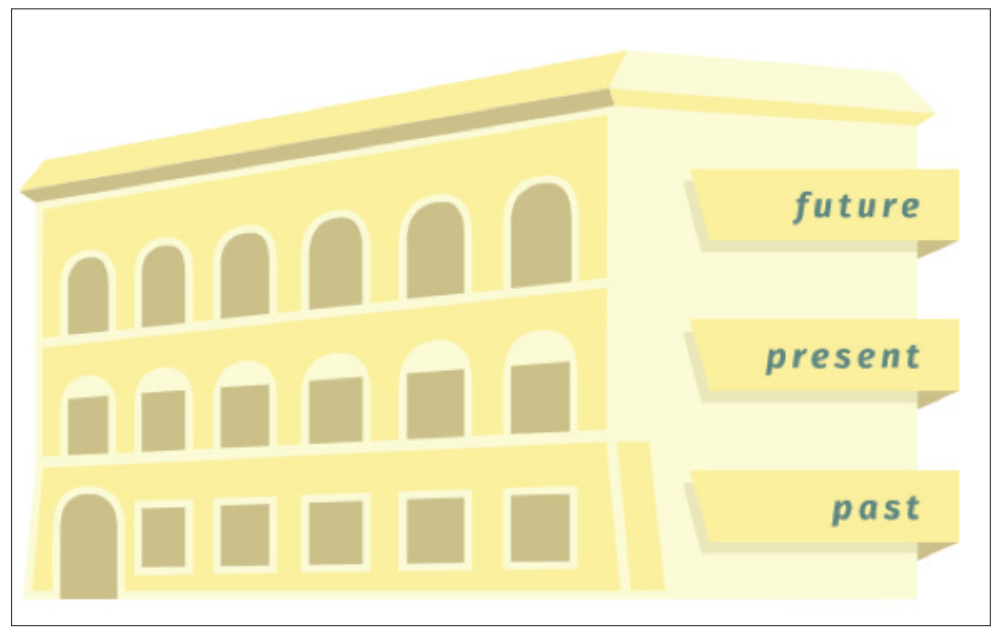

I tre piani della

"Casa della responsabilità".

R. Stammler, Hitler-Museum in Braunau? Idee löst im Innviertel viel Kopfschütteln aus, «Oberösterreichische Nachrichten», 8 settembre 2017. Waidbacher si riferisce al paragrafo 1 della legge, in base al quale si deve impedire ogni «promozione o promulgazione dell'ideologia nazionalsocialista».

16 T. Sendlhofer, Hitlers Geburtshaus: Minister Kickl steht hinter Plänen, «Der Kurier», 6 aprile 2018. 
dedicato al futuro, provando a formulare soluzioni per un mondo globalizzato e caratterizzato dalla coabitazione di persone provenienti da paesi diversi. La "Casa della Responsabilità" quindi non diventerebbe un luogo focalizzato sulla storia locale o un centro di documentazione come il vicino Obersalzberg in Baviera, ma un luogo dove si parla della responsabilità collettiva per il presente $\mathrm{e}$ il futuro tramite una riflessione critica sul passato, che coinvolga soprattutto le giovani generazioni ${ }^{17}$.

\section{Bibliografia}

Baumhackl U. 2015, Das Geisterhaus. Wo Hitler geboren wurde - eine Immobilie, die keiner will, in Götz T. e Winkler S. (eds.) 2015, Narben des Krieges. Von Braunau bis Jalta. 19 Reisen zu Orten, die der Zweite Weltkrieg gezeichnet hat, Graz.

Judt T. 2002, The past is another country: Myth and Memory in post-war Europe, in Müller J.-W. (ed.) 2002, Memory and Power in post-war Europe. Studies in the presence of the past, Cambridge.

Liebhart K. 2007, Politisches Gedächtnis und Erinnerungskultur - Die Bundesrepublik Deutschland und Österreich im Vergleich, in Gehler M. e Böhler I. (eds.) 2007, Verschiedene europäische Wege im Vergleich. Österreich und die Bundesrepublik Deutschland, 1945/49 bis zur Gegenwart, Innsbruck / Wien / Bozen.

Schöndorfer T. 2018, Expropriation of Adolf Hitler's Birthplace: Justified under the Special Historic Responsibility of Austria, «ICL Journal», 12, 1.

Uhl H. 2001, Das 'erste' Opfer. Der österreichische Opfermythos und seine Transformationen in der Zweiten Republik, "Österreichische Zeitschrift für Politikwissenschaft", 30,1 .

\section{Risorse on line}

Österreichischer Auslandsdienst - Austrian Service Abroad www.auslandsdienst.at

Informazioni e documenti sulla "Braunau-History" http://braunau-history.at/w/index.php?title=Geburtshaus

Comunicazione stampa della Corte Suprema sulla costituzionalità dell'esproprio della casa natale di Hitler https://www.vfgh.gv.at/medien/Enteignung_von_Hitlers_Geburtshaus.de.php 
Progetto Haus der Verantwortung ("Casa della responsabilità") http://www.hrb.at/

Articolo di Melissa Eddy, In Adolf Hitler's Hometown, Trying to Overcome a Legacy of Evil, «New York Times», 11 febbraio 2015 https://www.nytimes.com/2015/02/12/world/europe/in-adolf-hitlers-hometown-tryingto-overcome-a-legacy-of-evil.html 